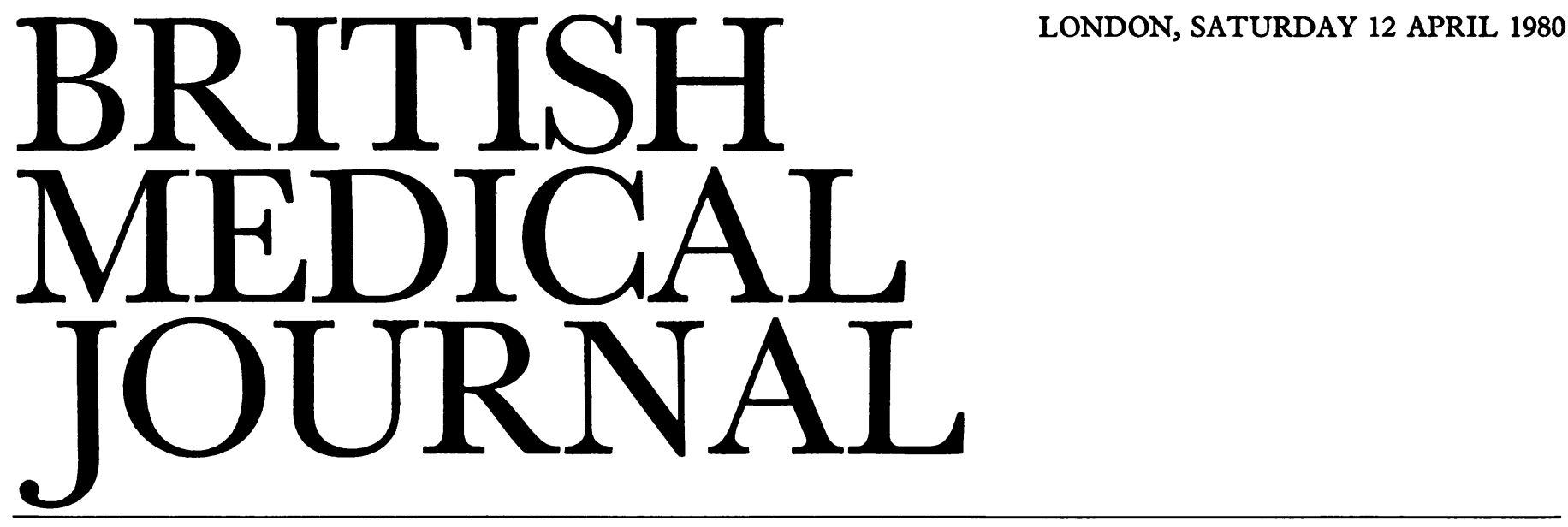

Correcting serious haemodynamic embarrassment in patients with acute circulatory disorders depends on accurate clinical assessment and appropriate measurements of intravascular pressures. Treatment is usually aimed at achieving a normal cardiac output and relieving or preventing pulmonary oedema. Safe and reliable techniques are now available for haemodynamic monitoring of these patients at the bedside. ${ }^{1}$

Within limits, the cardiac output increases as the ventricular filling pressure (or atrial pressure) rises. ${ }^{2} 3$ One important determinant of atrial pressure is plasma volume, and in lowoutput states due to haemorrhage or surgery transfusion of whole blood will restore the cardiac output to normal. But as the left atrial pressure rises above $20 \mathrm{mmHg}$ (with reference to mid-chest) pulmonary oedema will develop, and this risk places a critical upper limit on the volume of transfusion that these patients will tolerate. Direct measurement of the left atrial pressure is a procedure for the catheter laboratory only, but the right atrial pressure can be measured easily with a central venous line attached to a manometer.

In the absence of cardiovascular disease the atrial pressures on the two sides of the heart are closely related ${ }^{4}$ and treatment by fluid replacement may therefore be titrated against right atrial pressure to avoid the risk of pulmonary oedema. Similarly, in patients with an overloaded circulation a central venous line facilitates an appropriate reduction of the plasma volume-by venesection, for example-so that pulmonary oedema may be cleared without an undesirable fall in atrial pressure and cardiac output. In patients whose haemodynamic problems are due solely to abnormalities in the plasma volume the aim of treatment should be right atrial pressure of 5-8 mm $\mathrm{Hg}$ (equivalent to a left atrial pressure of $9-13 \mathrm{~mm} \mathrm{Hg}$ ). In the absence of cardiovascular disease no advantage is gained from increasing atrial pressures above the physiological range.

When, however, the normal balance between left and right ventricular function is disrupted-as may occur in ischaemic heart disease-the predictable relation between left and right atrial pressures is lost, and measurement of one provides no useful information about the other. ${ }^{6}$ Changes in atrial pressure may be due to alterations in venous tone, ventricular compliance, and ventricular contractility resulting in shifts in the distribution of blood between the systemic and pulmonary circulations, since a rise in the filling pressure of the stressed ventricle will help to maintain its output. If left ventricular disease underlies the haemodynamic disturbance and perfusion of vital organs becomes threatened by the low cardiac output, knowing the left atrial pressure may be essential for appropriate management. A central venous line cannot provide this information, but an indirect measure of left atrial pressure may be made at the bedside with a balloon-tipped catheter attached to a pressure transducer. ${ }^{7}$ When the catheter is introduced into an antecubital or central vein inflation of the balloon allows it to be flow-guided through the right side of the heart into the pulmonary artery (without fluoroscopy). By this simple means the end-diastolic pressure in the pulmonary artery may be measured, and in most circumstances this provides a reliable index of left atrial pressure. ${ }^{8}$

Monitoring the pressure in the pulmonary artery finds its major application in the management of patients with severe left ventricular dysfunction during and after major surgical procedures or with life-threatening pump failure complicating myocardial infarction. In both groups of patients increasing the left ventricular filling pressure provides one means of improving the cardiac output, but unless treatment is guided by measurements of the end-diastolic pressure in the pulmonary artery pulmonary oedema may be precipitated.

Patients with left ventricular disease recovering from surgery often depend on a high left atrial pressure to maintain cardiac output and renal perfusion. Thus plasma volume is critical, and insistence on inflexible fluid regimens or the use of diuretics to promote the flow of urine may be dangerous if the pulmonary artery end-diastolic pressure is not known.

When severe left ventricular failure complicates acute myocardial infarction the left atrial pressure is usually considerably raised and the prognosis is poor. A pulmonary artery catheter is a useful aid to management, expecially when early treatment does not produce a prompt response. If renal perfusion is satisfactory treatment with a diuretic will often reduce the left atrial pressure and allow pulmonary oedema to clear. Over-diuresis producing an excessive reduction of plasma volume should be avoided, since this may lower the left atrial pressure and the cardiac output. Careful haemodynamic monitoring allows an optimal end-diastolic pressure of 14-18 $\mathrm{mm} \mathrm{Hg}$ to be maintained in the pulmonary artery. ${ }^{9}$ If the cardiac output is already critically low diuretics are rarely helpful. In these circumstances vasodilators such as glyceryl trinitrate may be used to reduce left atrial pressure by their action on venous tone. ${ }^{10}$ Many drugs of this type-for example, nitroprusside ${ }^{11}$ -have a similar action on arteriolar tone, and in heart failure 
their use causes the cardiac output to increase by reducing the resistance to ventricular ejection. Vasodilator drugs have extremely potent cardiovascular effects, however, and patients who are acutely ill need haemodynamic monitoring to derive the greatest benefit from this form of treatment. In a small but important group of patients with low-output states complicating myocardial infarction the left atrial pressure is normal or even low. ${ }^{12}$ Again, measurement of the pulmonary artery enddiastolic pressure is necessary to identify these patients, who usually respond well to expansion of the plasma volume.

Applying these principles, then, will facilitate the management of patients with acute haemodynamic disorders. In small groups of patients there has also been evidence that this approach may improve survival rates, at least in the short term ${ }^{13} 14$ - a possibility that calls for further study.

1 Armstrong PW, Baigrie RS. Hemodynamic monitoring in the critically ill patient : overview of symposium. Can Med Assoc F 1979;121:865-6.

2 Braunwald E, Frahm CJ, Ross J Jr. Studies on Starling's law of the heart. V Left ventricular function in Man. F Clin Invest 1961;40:1882-90.

3 Bishop VS, Stone HL, Guyton AC. Cardiac function curves in conscious dogs. Am $\mathcal{F}$ Physiol 1964 ;207:677-82.

4 Berglund E. Ventricular function VI. Balance of left and right ventricular output: relation between left and right atrial pressures. Am $\mathcal{F}$ Physiol 1954;178:381-6

5 Cohn JN, Tristani FE, Khatri IM. Studies in clinical shock and hypotension VI. Relationship between left and right ventricular function. f Clin Invest 1969;48:2008-18.

6 Forrester JS, Diamond G, McHugh TJ, Swan HJC. Filling pressures in the right and left sides of the heart in acute myocardial infarction. A reappraisal of central venous pressure monitoring. N Engl f Med 1971; 285:190-3.

7 Swan HJC, Ganz W, Forrester JS, Marcus H, Diamond G, Chonette D. Catheterization of the heart in man with use of flow-directed balloontipped catheter. $N$ Engl $\mathcal{F}$ Med $1970 ; 283: 447-51$.

8 Jenkins BS, Bradley RD, Branthwaite MA. Evaluation of pulmonary arterial end-diastolic pressure as an indirect measure of left atrial mean pressure. Circulation 1970;42:75-8.

- Crexells C, Chatterjee K, Forrester JS, Dikshit K, Swan HJC. Optimal level of filling pressure in the left side of the heart in acute myocardial infarction. $N$ Engl f Med 1973 ;289:1263-6.

${ }^{10}$ Delgado CE, Pitt B, Taylor DR, Weisfeldt ML, Kelly DT. Role of sublingual nitroglycerin in patients with acute myocardial infarction. Br Heart f 1975 ;37:392-6.

11 Chatterjee K, Parmley WW, Ganz W, et al. Hemodynamic and metabolic responses to vasodilator therapy in acute myocardial infarction. Circulation $1973 ; 48: 1183-93$.

12 Forrester JS, Diamond G, Chatterjee K, Swan HJC. Medical therapy of acute myocardial infarction by application of hemodynamic subsets. $N$ Engl f Med 1976 ;295:1404-13.

13 Chatteriee K, Swan HJC, Kaushik VS, Joblin G, Magnusson P, Forrester JS. Effect of vasodilator therapy for severe pump failure in acute myocardial infarction on short-term and late prognosis. Circulation $1976 ; 53: 797-802$.

14 Fowler MB, Timmis AD, Chamberlain DA. Synergistic effects of a combined salbutamol-nitroprusside regimen in acute myocardial infarction and severe left ventricular failure. $\mathrm{Br}$ Med $\mathcal{F}$ 1980; 280:435-7.

\section{Early diagnosis of carcinoma of the prostate}

The quest for early diagnosis in any condition is founded on the belief that intervention at that stage will produce a higher survival rate and better quality of life for the patient. The degree to which treatment influences prognosis in early carcinoma of the prostate is still, however, uncertain-as is the form it should take: radical surgery, orchidectomy, hormonal therapy, and radiotherapy all have their champions.

Because of this uncertainty a Medical Research Council trial has been set up to evaluate the place of radiotherapy in the treatment of early $\left(T_{0}\right.$ and $\left.T_{1}\right)$ prostatic tumours without metastases; a further MRC trial is to compare orchidectomy, radiotherapy, and a combination of both in the treatment of larger $\left(T_{2}, T_{3}\right.$, and $\left.T_{4}\right)$ prostatic tumours without metastases. There is, however, a growing body of opinion that early carcinoma of prostate may be cured by either radical surgery or radiotherapy. The effect of treatment on survival is particularly difficult to assess in carcinoma of prostate, which may be undiagnosed in so many cases. The increased effectiveness of modern diagnosis may be uncovering patients with an excellent prognosis, so creating a spurious impression of improved survival. Carefully controlled clinical trials are needed to resolve the uncertainties ${ }^{1}$; but meanwhile every effort should be made to establish the diagnosis as soon as possible, and for that reason some new biochemical approaches are provoking wide interest.

The time-honoured reliance by the clinician on digital palpation of the prostate has become unsatisfactory as radical methods of treatment have made precise diagnosis mandatory. The cornerstone of accurate diagnosis lies in histological examination. The needle biopsy, which can be performed so effectively with the Tru-cut needle by the transrectal or transperineal route, allows accurate diagnosis and even a small nodule can be precisely sampled. Less satisfactory is the aspiration biopsy, ${ }^{2}$ which provides only a cytological sample; diagnosis is less reliable, as interpretation can be difficult even for a skilled cytologist.

Even more unreliable are biochemical aids such as estimation of the acid phosphatase, in particular its tartrate labile form, sometimes termed the "prostatic" component. High values certainly indicate the presence of bony metastases, but the concentration is frequently normal when disease is confined to the prostate, and technetium bone scans have shown that bony metastases are often present in patients with normal bone radiographs and normal concentrations of acid phosphatase. ${ }^{3}$ In a comparison of three enzymic methods of estimation with a double antibody radioimmunoassay, ${ }^{3}$ the latter gave more positive results but yielded high values in only $8 \%$ of the patients with intracapsular disease with no metastases. False-positive results can also occur, though the longstanding belief that prostatic digital examination raised the phosphatase concentration even in the normal patient has been largely discredited. ${ }^{4}$

A screening test for early malignant change in the prostate has long been sought, and a possible basis is the recognised biochemical phenomenon of a lower zinc concentration in malignant prostatic tissue than in benign hypertrophy or in the normal gland. ${ }^{5}$ Another discriminant is the amount of testosterone in prostatic tissue; concentrations are higher in carcinoma of the prostate than in benign hypertrophy. ${ }^{\circ}$ Habid $e l a l^{7}$ have recently suggested that estimating the zinc and hormone concentrations may provide a reliable test for distinguishing between benign and malignant disease in the prostate. Any change in the zinc content of the gland, however, is likely to result from the development of a frank carcinoma and not to precede it. No one has yet shown a change in zinc content except as a result of a longstanding tumour. Habid and his co-workers used transurethral resection specimens, and in as many as $20 \%$ of resections these yield benign tissue even when a definite neoplasm exists in the periphery of the gland. Clearly, therefore, if the prostatic zinc content is found to be low even when carcinoma is confined to the periphery of the gland this change could be a valuable marker. Reduced zinc concentrations have been reported in the leucocytes of patients with liver disease, ${ }^{8}$ but whether any similar alteration occurs in prostatic disease is not yet known. 